\title{
High density 3D printed microfluidic valves, pumps, and multiplexers
}

Hua Gong

Adam T. Woolley

Gregory P. Nordin

Brigham Young University - Provo, nordin@byu.edu

Follow this and additional works at: https://scholarsarchive.byu.edu/facpub

Part of the Electrical and Computer Engineering Commons

\section{Original Publication Citation}

H. Gong, A. T. Woolley and G. P. Nordin, Lab Chip, 2016, DOI: 10.1039/C6LC00565A.

\section{BYU ScholarsArchive Citation}

Gong, Hua; Woolley, Adam T.; and Nordin, Gregory P., "High density 3D printed microfluidic valves, pumps, and multiplexers" (2016). Faculty Publications. 1656.

https://scholarsarchive. byu.edu/facpub/1656

This Peer-Reviewed Article is brought to you for free and open access by BYU ScholarsArchive. It has been accepted for inclusion in Faculty Publications by an authorized administrator of BYU ScholarsArchive. For more information, please contact ellen_amatangelo@byu.edu. 


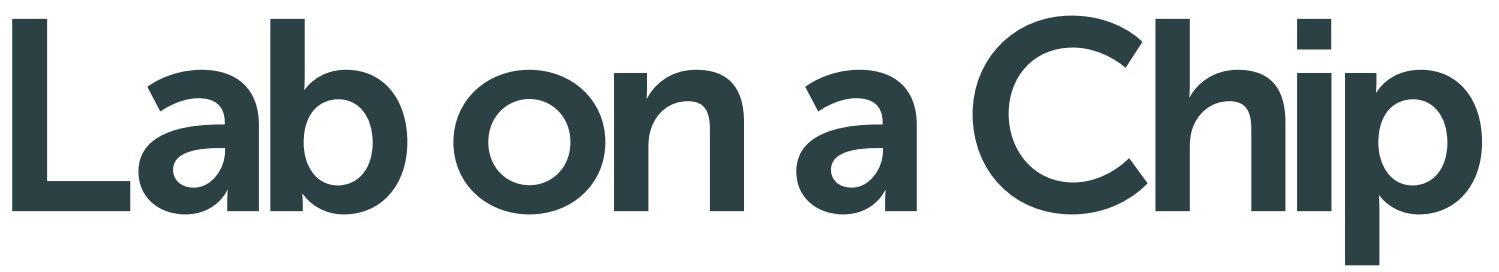

\section{Accepted Manuscript}

This article can be cited before page numbers have been issued, to do this please use: H. Gong, A. T. Woolley and G. P. Nordin, Lab Chip, 2016, DOI: 10.1039/C6LC00565A.

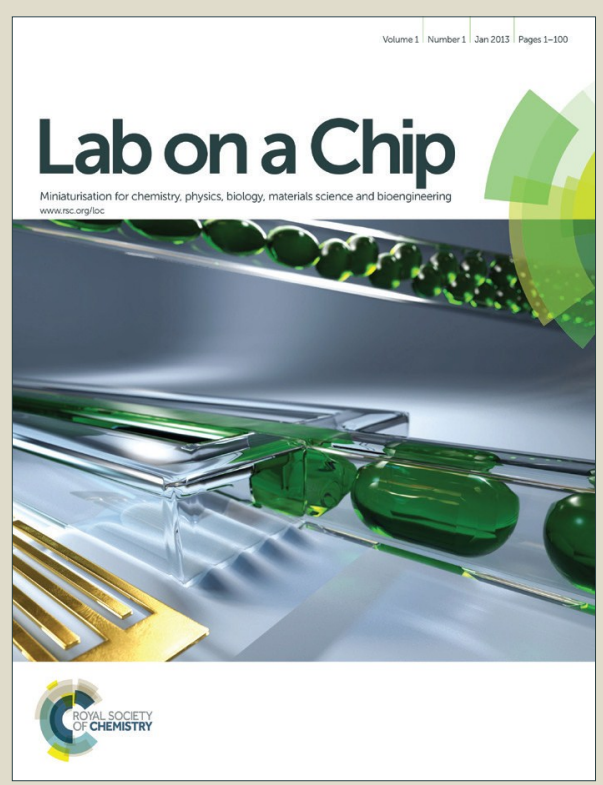

This is an Accepted Manuscript, which has been through the Royal Society of Chemistry peer review process and has been accepted for publication.

Accepted Manuscripts are published online shortly after acceptance, before technical editing, formatting and proof reading. Using this free service, authors can make their results available to the community, in citable form, before we publish the edited article. We will replace this Accepted Manuscript with the edited and formatted Advance Article as soon as it is available.

You can find more information about Accepted Manuscripts in the Information for Authors.

Please note that technical editing may introduce minor changes to the text and/or graphics, which may alter content. The journal's standard Terms \& Conditions and the Ethical guidelines still apply. In no event shall the Royal Society of Chemistry be held responsible for any errors or omissions in this Accepted Manuscript or any consequences arising from the use of any information it contains. 


\title{
Journal Name
}

\section{ARTICLE TYPE}

Cite this: DOI: $10.1039 / x x x x x x x x x x$

\section{High density 3D printed microfluidic valves, pumps, and multiplexers ${ }^{\dagger}$}

\author{
Hua Gong, ${ }^{a}$ Adam T. Woolley, ${ }^{b}$ and Gregory P. Nordin ${ }^{\star a}$
}

Received Date

Accepted Date

DOI: 10.1039/xxxxxxxxxx

www.rsc.org/journalname

In this paper we demonstrate that 3D printing with a Digital Light Processor stereolithographic (DLP-SLA) 3D printer can be used to create high density microfluidic devices with active components such as valves and pumps. Leveraging our previous work on optical formulation of inexpensive resins (RSC Adv. 5, 106621, 2015), we demonstrate valves with only $10 \%$ of the volume of our original 3D printed valves (Biomicrofluidics 9, 016501, 2015), which were already the smallest that have been reported. Moreover, we show that inclusion of a thermal initiator in the resin formulation along with a post-print bake can dramatically improve the durability of $3 \mathrm{D}$ printed valves up to 1 million actuations. Using two valves and a valve-like displacement chamber (DC), we also create compact 3D printed pumps. With 5-phase actuation and a $15 \mathrm{~ms}$ phase interval, we obtain pump flow rates as high as $40 \mu \mathrm{L} / \mathrm{min}$. We also characterize maximum pump back pressure (i.e., maximum pressure the pump can work against), maximum flow rate (flow rate when there is zero back pressure), and flow rate as a function of the height of the pump outlet. We further demonstrate combining 5 valves and one DC to create a 3-to-2 multiplexer with integrated pump. In addition to serial multiplexing, we also show that the device can operate as a mixer. Importantly, we illustrate the rapid fabrication and test cycles that 3D printing makes possible by implementing a new multiplexer design to improve mixing, and fabricate and test it within one day.

\section{Introduction}

As we observed in Ref. 1, 3D printing offers a true rapidprototyping capability for microfluidics in which a "fail fast and often" strategy can be employed to positively disrupt the microfluidic device development process by using early and rapid empirical feedback to iteratively guide and accelerate device development. A key enabler of rapid iteration is fast 3D printing and post-processing such that one or more iterations of fabrication $\rightarrow$ test $\rightarrow$ adjust design can be performed in a single day. In addition to a vastly streamlined fabrication process, 3D printing avoids the significant startup and operational costs of a cleanroom, thereby making microfluidic device development available to a much broader audience. Moreover, use of standardized elements and functional blocks in the design and layout of a device can permit non-specialists to apply microfluidics to research, prototyping, and product development in many fields. In our judgment, these factors are indicative of the exceptional potential 3D

\footnotetext{
a Department of Electrical and Computer Engineering, Brigham Young University, Provo, UT 84602, United States; E-mail: nordin@byu.edu

${ }^{b}$ Department of Chemistry and Biochemistry, Brigham Young University, Provo, UT 84602, United States.

$\dagger$ Electronic Supplementary Information (ESI) available. See DOI: $10.1039 / \mathrm{b} 000000 \mathrm{x} /$
}

printing offers ${ }^{2-4}$ to effect a step-change in the trajectory of microfluidics usage similar to the transition in the late 1990's from glass and silicon microfluidics to soft lithography ${ }^{5}$ using polydimethylsiloxane (PDMS). ${ }^{6,7}$

Despite this attractive vision for a much expanded microfluidics community, a number of misconceptions and deficiencies stand in the way of its fulfillment. For example, as we discussed in Ref. 8, microfluidic devices consist of a series of small interconnected voids within a bulk material, and traditional commercial 3D printing tools and resins are not optimized for fabrication of such structures. We demonstrated that with proper formulation of resin optical properties, flow channels with cross sections as small as $60 \mu \mathrm{m} \times 108 \mu \mathrm{m}$ could be reliably fabricated with a commercial digital light processing stereolithographic (DLP-SLA) 3D printer. The resin optical properties primarily affect the vertical (out-of-plane) build resolution, while the DLP pixel pitch and optical imaging system are the critical factors for in-plane resolution.

There is a common misperception that the manufacturerspecified 3D printer resolution represents the achievable void feature size that can be fabricated with the printer. However, as we showed in Ref. 8, this is not the case. The minimum void height is determined by the optical absorption of the resin (we 
found experimentally that it is $\sim 3.5-5.5 h_{a}$ where $h_{a}$ is the inverse of the resin absorption coefficient), while the minimum reliable lateral dimension is 4 times the pixel pitch in the build plane. Nonetheless, some impressive work has been done with commercially available 3D printing tools and materials, including reactionware devices, ${ }^{9,10}$ snap-together discrete microfluidic elements, ${ }^{11}$ a continuous nitrate-monitoring device for water contamination, ${ }^{12}$ mail-order microfluidics using a commercial service bureau, ${ }^{13}$ active devices (valves and pumps), ${ }^{14}$ and microfluidic circuitry (analogous to electronic circuitry). ${ }^{15}$ The primary disadvantages of these approaches to date is the relatively large minimum void size and consequent overall device size.

In this paper we show significant miniaturization of 3D printed microfluidic devices with integrated valves and pumps based on our previous resin formulation work ${ }^{8}$ and our demonstration of the first reported 3D printed valves. ${ }^{1}$ Specifically, we show how to use a DLP-SLA 3D printer with our inexpensive custom resin formulation to fabricate robust membrane valves 40 pixels in diameter $(1.08 \mathrm{~mm})$ with a minimum chamber height of $60 \mu \mathrm{m}$. These valves are only $10 \%$ the volume of our previous 3D printed valves, ${ }^{1}$ and we have improved their durability from 800 actuations to 1 million actuations. To achieve such durability, we modify the resin composition by adding a thermal initiator such that a post-printing 30 minute oven cure drives further polymerization of the material to create a greater degree of cross linking and mechanical toughness. We then demonstrate a simple pump structure consisting of two valves and one displacement chamber (DC), and experimentally characterize its maximum back pressure and maximum flow rate. Finally, we combine 5 valves and one DC into a compact 3-to-2 multiplexer with integrated pump, utilizing the flexibility of 3D printing to densely arrange device elements within the 3D volume of the device. We also show that the multiplexer can be used as a mixer and that its mixing efficiency can be improved by increasing the number of inlets in the DC.

\section{Experimental}

\subsection{Materials}

Our resin formulations consist of monomer, photoinitiator, and absorber, which for this work are poly(ethylene glycol) diacrylate (PEGDA, MW 258), Sudan I, and Irgracure 819, respectively. ${ }^{1,16,17}$ We also include a thermal initiator, azobisisobutyronitrile (AIBN), for post-print thermal curing, the details of which are discussed in Sect. 3.1. It is important to note that use of a low molecular weight PEGDA results in excellent water stability for fabricated parts, ${ }^{16}$ with no swelling or degradation in mechanical strength. PEGDA, Sudan I, and AIBN are obtained from Sigma-Aldrich (St. Louis, MO), and Irgacure 819 from BASF (Vandalia, Illinois). Each is used as received.

The specific resin formulation employed for the work reported in this paper is the $0.4 \%$ Sudan I resin discussed in Ref. 8 . It is prepared by mixing $0.4 \%(w / w)$ Sudan I, 1\% (w/w) Irgacure 819, and $0.01 \%(\mathrm{w} / \mathrm{w})$ AIBN with PEGDA, and sonicating for 30 minutes. The resin is stored in an amber glass bottle wrapped in aluminum foil to protect it from light exposure.

\subsection{D printer}

We use an Asiga Pico Plus 27 DLP-SLA 3D printer as described in Ref. 8, which has an LED peak wavelength of $412 \mathrm{~nm}$ and an in-plane resolution (pixel pitch) of $27 \mu \mathrm{m}$. Microfluidic devices in an individual print run are fabricated on a glass slide $(25 \mathrm{~mm}$ x $37.5 \mathrm{~mm} \times 1.2 \mathrm{~mm}$ ) which is attached to the printer build table with double-sided tape. We experienced no issues with the slide damaging the teflon film comprising the bottom of the resin tray as long as we followed the 3D printer manufacturer's build table alignment procedure. Each slide is prepared by cleaning with acetone and isopropyl alcohol (IPA), followed by immersion in $2 \%$ 3-(trimethoxysilyl) propyl methacrylate in toluene for 2 hours. After silane deposition slides are kept in toluene until use.

There are two reasons we use glass slides. The first is that they avoid the need to fabricate the first device layer on the rough (anodized Al) surface of the 3D printer build table, which, especially for resins with high optical absorbance, requires a significantly longer exposure time for the first layer to attach to the build table. Long exposure times deplete the available binding sites on the surface of the layer, making attachment of the next layer problematic. The second reason is that the smooth surfaces of the glass slide offer convenient optical access to observe the interior components of the microfluidic device.

\subsection{Device fabrication}

Our build layer thickness, $l$, is $10 \mu \mathrm{m}$, which results in a normalized layer thickness, $\zeta=l / h_{a}$, of 0.57 for the $0.4 \%$ Sudan I resin. This is well within the optimal build thickness range we established in Ref. 8.

The key active component in our devices is a membrane valve, the structure of which is shown in Fig. 1a. ${ }^{1}$ The valve consists of a $20 \mu \mathrm{m}$ thick membrane (i.e., 2 build layers) sandwiched between two cylindrical voids which comprise a control chamber (100 $\mu \mathrm{m}$ high) and a fluid chamber (60 $\mu \mathrm{m}$ high), each 40 pixels $(1.08 \mathrm{~mm})$ in diameter. The corresponding dimensions of our original 3D printed valves are $50 \mu \mathrm{m}$ membrane thickness, with $250 \mu \mathrm{m}$ control chamber and $250 \mu \mathrm{m}$ fluid chamber heights, both of which are $2 \mathrm{~mm}$ in diameter. ${ }^{1}$ The valves in this paper are only $10 \%$ of the volume of the valves in our original paper $\left(0.165 \mathrm{~mm}^{3}\right.$ compared to $1.73 \mathrm{~mm}^{3}$ ). The valves in our original paper were fabricated with a different DLP-SLA 3D printer (B9 Creator) prior to developing our quantitative approach to resin formulation. ${ }^{8}$

When no pressure is applied to the control chamber (as illustrated in Fig. 1b), the valve is open and fluid can flow between the two channels at the bottom of the fluid chamber. A photomicrograph of an open valve is shown in Fig. 1d. The lighting makes it easy to see the pixelation of the bottom surface of the fluid chamber. The measured surface roughness for horizontal surfaces fabricated with $0.4 \%$ Sudan I resin is $0.5 \mu \mathrm{m}$ with a length scale the size of the pixel pitch. As shown in Fig. 1c, when pressure is applied to the control chamber the membrane deflects downward and seals the fluid channels. The central circular region in which the membrane contacts the bottom of the fluid chamber can be clearly seen in Fig. 1e.

In our devices valves are connected with flow channels that 
are $150 \mathrm{um}$ high and 6 pixels $(162 \mu \mathrm{m})$ wide. Although smaller channels can be fabricated with $0.4 \%$ Sudan I resin, ${ }^{8}$ we chose this somewhat larger size to ensure easy, high yield fabrication, even for flow channels as long as $118 \mathrm{~mm}$ (for a design that is not included in this paper). Consequently, we never had a problem with flow channel fabrication for the devices discussed in subsequent sections.

After 3D printing, unpolymerized resin must be flushed from the interiors of microfluidic structures. An advantage of our PEGDA resin formulations is that they are low viscosity $(57 \mathrm{cP}){ }^{8}$ making flushing much easier than for many commercial resins that have significantly higher viscosity. As shown in Ref. 8, the difficulty of flushing unpolymerized resin can be the limiting factor in determining the minimum achievable void size for commercial resins, rather than the actual optical properties of the resin.

Immediately after 3D printing is completed, the sample is removed from the build table and soaked in IPA for 2-3 minutes to dissolve much of the unpolymerized resin. The control and fluid chambers are then carefully flushed with IPA, following which vacuum is applied to extract residual IPA from microfluidic structures. Note that the control chamber design in Fig. 1a has a second channel specifically to permit flushing unpolymerized resin.

Next, we bake the device at $80^{\circ} \mathrm{C}$ for 30 minutes to activate the thermal initiator to drive further polymerization. It should be noted that a blanket UV exposure is completely ineffective at curing our 3D printed devices because the UV light only penetrates a small fraction of a millimeter even after many hours of exposure due to the high absorption of the resin.

After baking, a device is prepared for testing by inserting Microbore PTFE tubing $\left(0.022^{\prime \prime} \mathrm{ID} \times 0.042^{\prime \prime} \mathrm{OD}\right)$ into corresponding cylindrical holes in the device (Fig. S1, $\mathrm{ESI}^{\dagger}$ ), and gluing the tubing in place (Loctite Instant Mix 5 Minute Epoxy). The epoxy is also used to plug the flushing channels for all control chambers.

Total device build and preparation time is approximately 4 hours, and consists of (1) 3D printing ( $<1$ hour), (2) flushing unpolymerized resin (5-7 minutes for all of the devices in a single print run), (3) oven bake (30 minutes), and (4) inserting tubing followed by mixing and applying epoxy to the junction between the tubing and device and letting the epoxy cure for 2 hours. Despite being labelled as 5 minute epoxy, we found that a 2 hour cure time was needed for the epoxy to achieve a hardness that facilitated leak-tight seals and good tubing strain relief. The epoxy cure step is by far the most time-consuming aspect of our current device fabrication and preparation process. This time can be dramatically reduced to just a few minutes by substituting a UV curable adhesive for the 5 minute epoxy, thereby reducing the total device fabrication and preparation time to under 2 hours.

CAD and .stl design files for the valve, pump, and multiplexer reported in this paper are freely available online. ${ }^{18}$

\section{Results and discussion}

\subsection{D printed valves}

To evaluate the performance of 3D printed valves, we first measured the maximum fluid pressure, $P_{\text {fluid }}$, at which the valve stays closed for a given control pressure, $P_{\text {control }}$, applied to the mem-

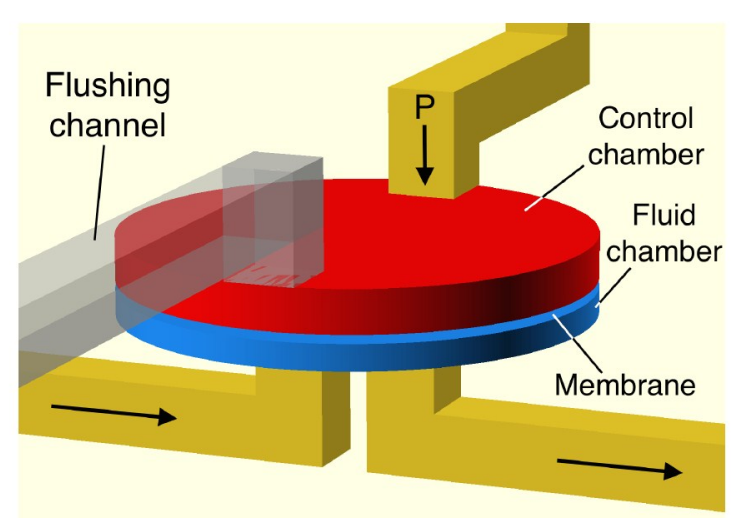

(a)

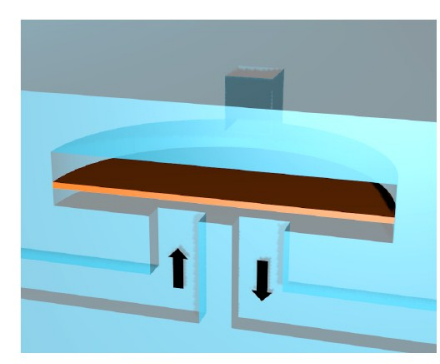

(b)

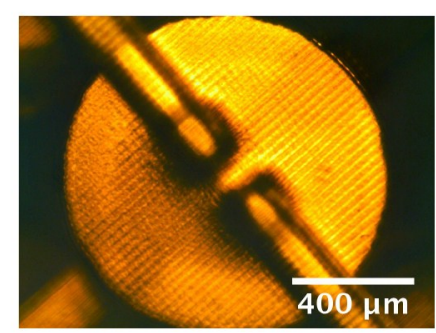

(d)

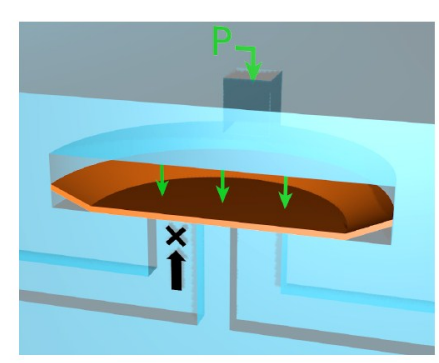

(c)

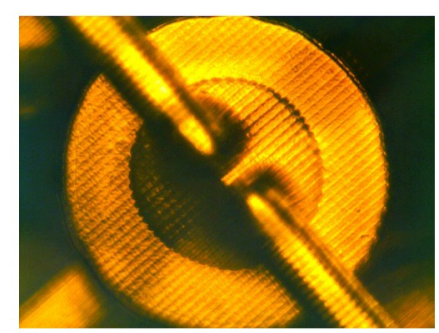

(e)
Fig. 1 (CAD design of (a) for a 3D printed membrane valve. Schematic illustration and microscope photos of (b), (d) open and (c), (e) closed valves. The microscope photos show the bottom of the valve. See text for details.

brane to close the valve. Measurement details are discussed in Section S2, $\mathrm{ESI}^{\dagger}$. The pressure difference, $\Delta P=P_{\text {control }}-P_{\text {fluid }}$, can be interpreted as the minimum pressure required to deflect the membrane enough to just barely close the valve. We therefore expect that $\Delta P$ should increase as the fluid chamber height increases or the membrane thickness increases because either change makes it more difficult to deflect the membrane enough to close the valve. This is confirmed in Figs. S2b, $\mathrm{ESI}^{\dagger}$ and S2c, $\mathrm{ESI}^{\dagger}$ which show the corresponding experimental measurements. In Fig. S2b, $\mathrm{ESI}^{\dagger}$, an increase in $\Delta \mathrm{P}$ from a little over $2 \mathrm{psi}$ to 4 psi is observed as the fluid chamber height increases from $50 \mu \mathrm{m}$ to $80 \mu \mathrm{m}$ because the membrane has to deflect more to close the valve. We experimentally determined that the fabrication yield for valves with a $50 \mu \mathrm{m}$ chamber height is substantially less than for a $60 \mu \mathrm{m}$ chamber height, which is nearly $100 \%$. We therefore chose a $60 \mu \mathrm{m}$ fluid chamber height for the devices reported in this paper. Similarly, as shown in Fig. S2c, ESI ${ }^{\dagger}$, the membrane 
becomes stiffer and requires more pressure to close as its thickness increases from 2 layers $(20 \mu \mathrm{m})$ to 4 layers ( $40 \mu \mathrm{m})$.

An important criterion for an active fluidic device is its durability. In our original demonstration of 3D printed valves, ${ }^{1}$ the valves typically lasted 800 actuations before the membranes broke. However, we had previously tested similar cleanroomfabricated valves to 115,000 actuations with no sign of breakage or degradation in their properties. ${ }^{17}$ Following 3D printing and prior to thermal curing, valve membranes in the present study typically lasted only one or two actuations before breaking. We hypothesized that additional polymerization was needed to increase the mechanical robustness of the membranes. However, as detailed in Ref. 8, other constraints prevent trying to achieve this with longer layer exposure times during $3 \mathrm{D}$ printing. Hence we added a thermal initiator with a post-print oven bake to drive further polymerization. We tested a variety of bake times at $80^{\circ} \mathrm{C}$, and found that they had no effect on pump properties as shown in Fig. S3, ESI ${ }^{\dagger}$. A bake time of 30 minutes seemed to offer the best trade-off between mechanically robust membranes, short bake time, and yield.

In our largest yield test, we began with 54 fabricated valves, one of which was found to leak between the flow channels during its first actuation. We later established that such as-built leaks can be avoided by simply increasing the separation between the channels in the bottom of the valve from 4 pixels to 6 pixels. Of the 53 remaining valves, all but one continued working after 10,000 actuations. The one that failed had its membrane break during the first 1,000 actuations. Of the 53 that survived, we tested one to 1 million actuations, after which it still worked. The flow channels and fluid chamber were filled with water during the entire 30 hours it took to run the test.

\subsection{D printed pumps}

As shown in Fig. 2a, a pump consists of two valves (V1 and V2) with a valve-like displacement chamber (DC) in between. The difference between a DC and a valve is that the inlet and outlet channels are placed on the edge of the fluid chamber for a DC rather than in the center as for a valve. This prevents the DC from being able to stop flow when actuated. Note also that the DC is oriented upside down compared to the valves. In a valve the fluid chamber is under the membrane whereas in the DC it is on top of the membrane.

Valve actuation is controlled by applying pressure to $\mathrm{C} 1$ and $\mathrm{C} 2$, whereas DC actuation is controlled with C3. A photo of a pump looking from underneath is shown in Fig. 2b. The DC appears to partially overlap V1 and V2 since they are placed at different heights in the 3D build volume. The undersides of cylindrical holes are also visible for PTFE tubing to connect C1, C2, and C3 to pressure sources, and the inlet and outlet channels to an offchip fluid source and sink. Figure $2 \mathrm{c}$ is a side view photo of a pump showing the fabricated spatial arrangement of the DC and valves, along with their corresponding flow and control channels.

The pump uses a 5-phase cycle as shown in Table 1 . At $t_{0}$, both valves are closed and pressure is applied to the DC. The DC fluid chamber is therefore in a state of minimum volume. At $t_{1}, \mathrm{~V} 1$

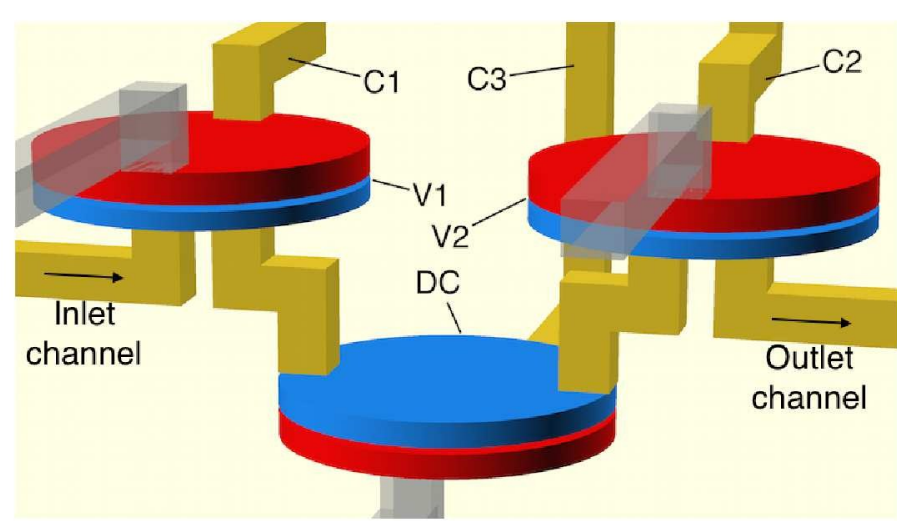

(a)

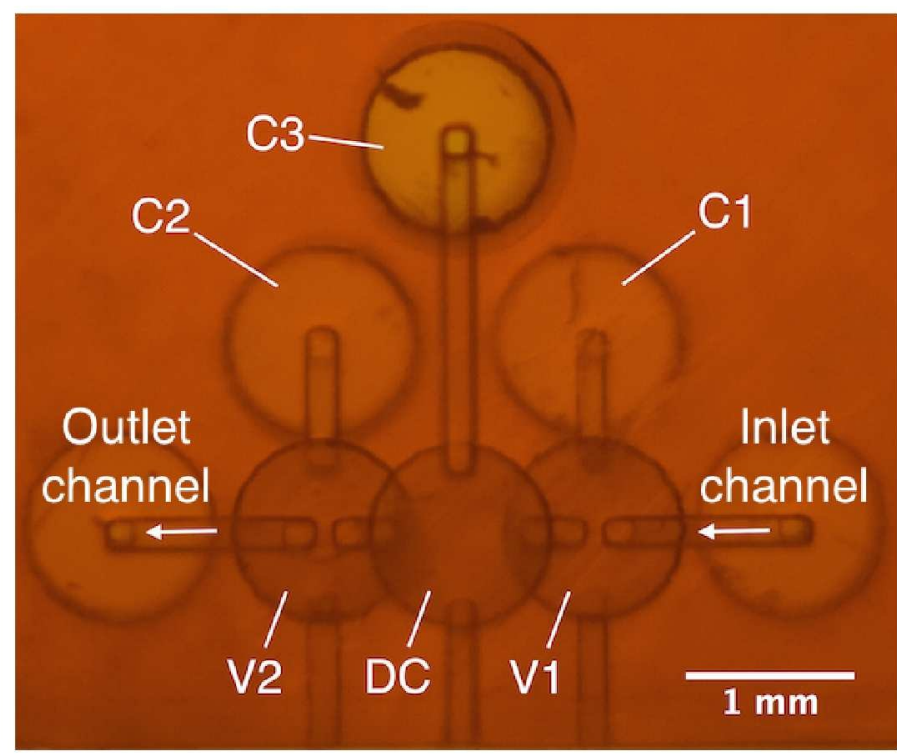

(b)

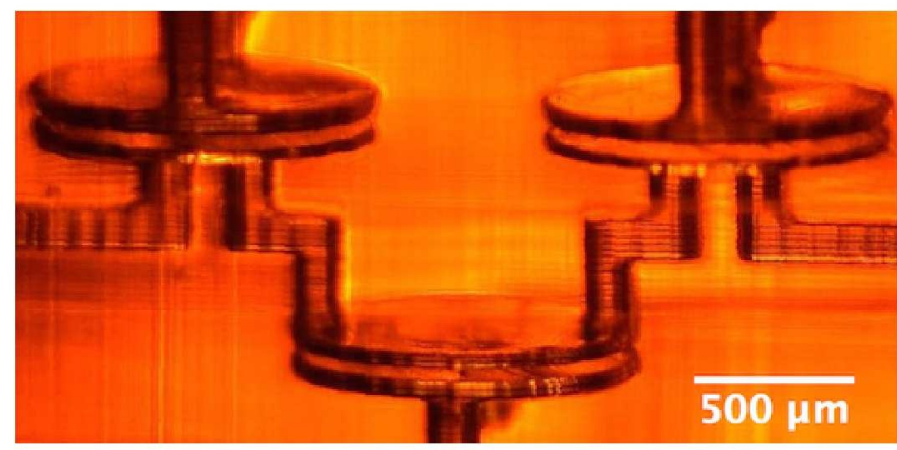

(c)

Fig. 2 (a) CAD design of 3D printed pump. C1-C3 connect to external pressure sources. The partially transparent channels are flushing channels for the valve control chambers, which are later sealed with epoxy. (b) Bottom view of a 3D printed pump. See text for details. (c) Side view photograph of 3D printed pump in (b).

opens and the DC pressure is released so the membrane returns to equilibrium thereby pulling fluid through V1 into the DC fluid chamber. At $t_{2}, \mathrm{~V} 1$ closes to isolate fluid in the DC from the inlet flow channel, followed by $\mathrm{V} 2$ opening at $t_{3}$ in preparation for 


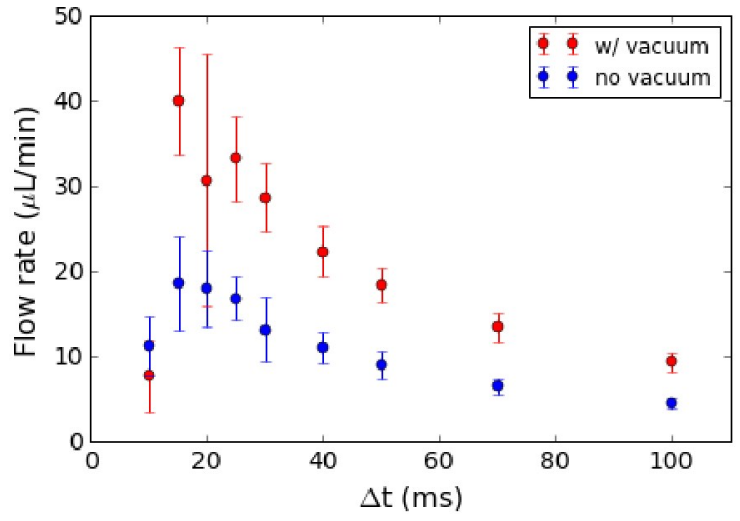

(a)

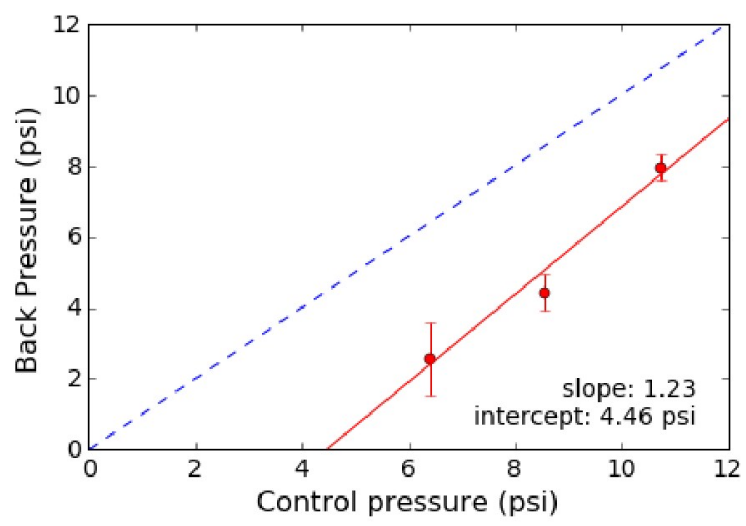

(c)

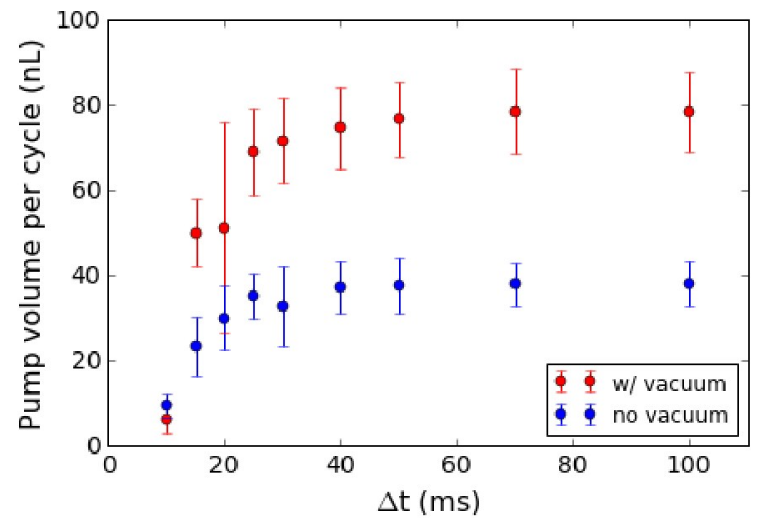

(b)

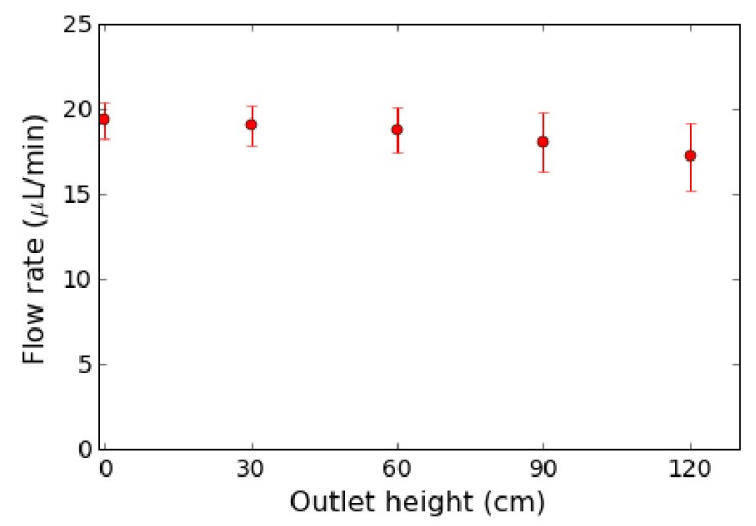

(d)

Fig. 3 (a) Maximum flow rate (zero back pressure) as a function of the phase interval, $\Delta t$. (b) Fluid volume expelled by pump for a single pump cycle calculated from the data in (a). 9 pumps were tested with vacuum and 5 without. The large error bars for vacuum at $20 \mathrm{~ms}$ are due to 2 pumps having significantly smaller flow rates than the others. (c) Maximum back pressure as a function of control pressure (average and standard deviation for 7 pumps). (d) Flow rate as a function of the outlet height for a control pressure of 9 psi (average and standard deviation for 6 pumps). Different pumps were used in all tests.

Table 1 Pump timing logic. Red: actuated (pressure applied; valves closed); green: not actuated (valves open).

\begin{tabular}{|c|c|c|c|c|c|}
\hline & $\mathrm{t}_{0}$ & $\mathrm{t}_{1}$ & $\mathrm{t}_{2}$ & $\mathrm{t}_{3}$ & $\mathrm{t}_{4}$ \\
\hline $\mathrm{V} 1$ & $\bullet$ & $\bullet$ & $\bullet$ & $\bullet$ & $\bullet$ \\
$\mathrm{DC}$ & $\bullet$ & $\bullet$ & $\bullet$ & $\bullet$ & $\bullet$ \\
$\mathrm{V} 2$ & $\bullet$ & $\bullet$ & $\bullet$ & $\bullet$ & $\bullet$ \\
\hline
\end{tabular}

expelling fluid from the DC into the outlet channel, which occurs by actuating the $\mathrm{DC}$ at $\mathrm{t}_{4}$. The cycle then repeats by going back to $t_{0}$ in which $\mathrm{V} 1$ is closed. The time difference between any two sequential phases is $\Delta t=t_{i}-t_{i-1}$, which we call the phase interval. Unless otherwise noted, the valve and DC actuation pressure is $\sim 9$ psi.

A pump's maximum flow rate, $Q_{\max }$, for a given set of operating parameters is defined as the flow rate when there is zero back pressure (such as when the fluid source, pump, and fluid outlet are all at the same height). We evaluated $Q_{\max }$ as a function of $\Delta \mathrm{t}$ under two conditions for the unactuated DC control chamber: (1) an applied vacuum of $10 \mathrm{psi}(520 \mathrm{mmHg})$ and (2) vent to at- mosphere. In the latter case the restoring force on the membrane is just the elastic strain of the stretched membrane. In the former, an applied vacuum at first assists the elastic strain until the membrane is undeflected and then pulls the membrane up into the control chamber.

The measured maximum flow rate, $Q_{\max }$, is shown as a function of $\Delta \mathrm{t}$ in Fig. 3a. The maximum flow rate increases as $\Delta t$ decreases, reaching a peak of $40 \mu \mathrm{L} / \mathrm{min}$ for $\Delta \mathrm{t}=15 \mathrm{~ms}$ when vacuum is applied to the $\mathrm{DC}$. The flow rate then drops rapidly as $\Delta \mathrm{t}$ continues to decrease. Note that for $\Delta \mathrm{t} \geq 15 \mathrm{~ms}$, the maximum flow rate with vacuum is approximately twice as large as without vacuum. Calculating the displaced fluid volume as a function of $\Delta \mathrm{t}$ (Fig. 3b), it is apparent that the vacuum pulls the membrane up into the control volume enough to essentially double the volume expelled by the DC for $t_{3} \rightarrow t_{4}$ as compared to the no vacuum case. We arbitrarily chose $50 \mathrm{~ms}$ (maximum expelled volume) as the phase interval for all subsequent measurements, and used vacuum with the DC.

A pump's maximum back pressure is defined as the maximum pressure the pump can work against such that the flow rate just goes to zero. Measurement details to determine a pump's maxi- 


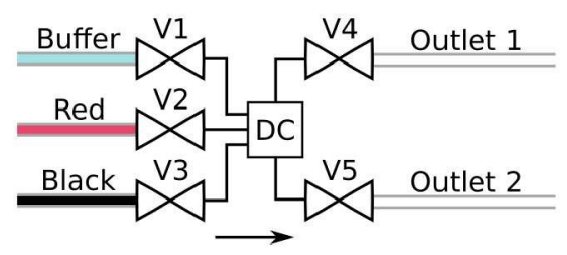

(a)

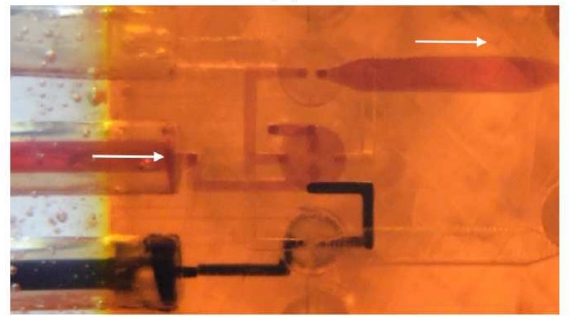

(d)

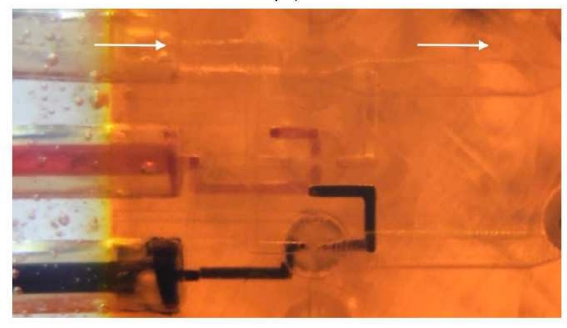

(g)

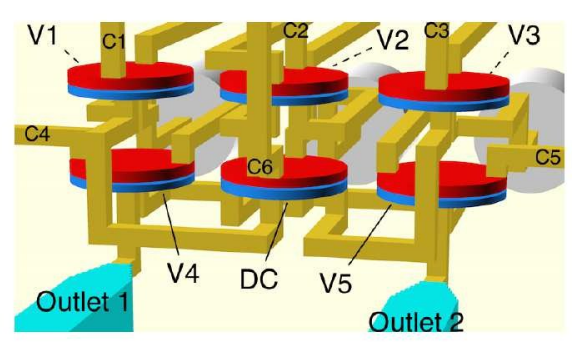

(b)

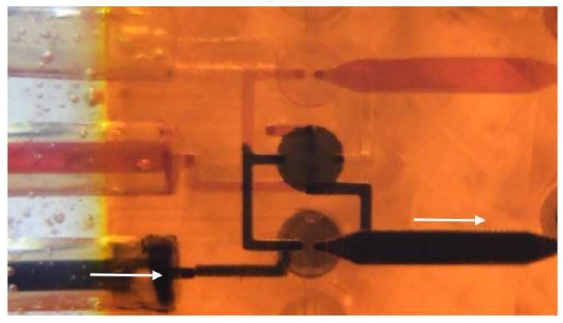

(e)

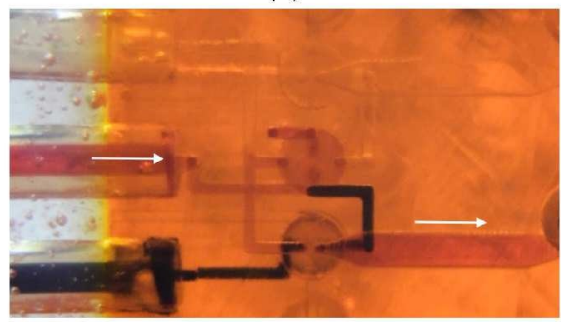

(h)

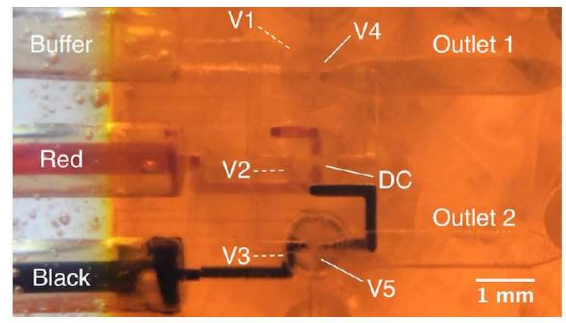

(c)

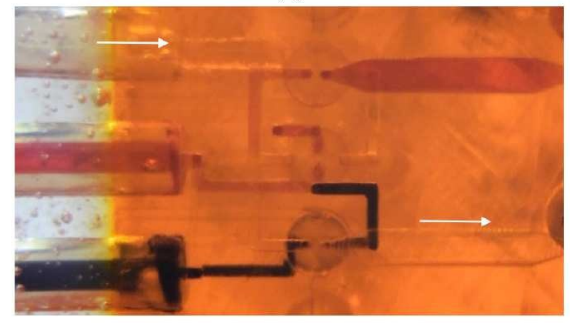

(f)

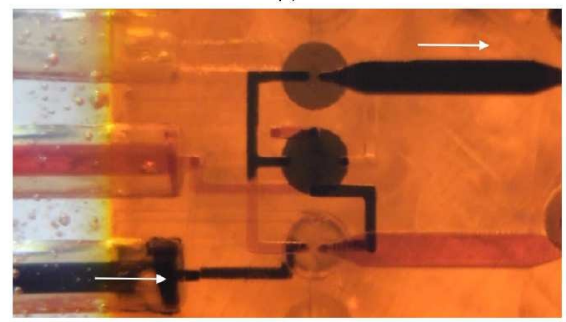

(i)

Fig. 4 (a) Multiplexer schematic diagram. (b) CAD design taking advantage of stacked layout flexibility enabled by 3D printing. Valve labeling is the same as (a) with corresponding control lines labeled C1, C2, etc. (c) Bottom view of multiplexer fabricated according to the CAD design in (b). (d)-(i) Demonstration of arbitrary 3-to-2 multiplexing. See text for details. Arrows indicate active flow direction.

mum back pressure are presented in Section S3, ESI ${ }^{\dagger}$, and a typical measurement is shown in Fig. S4, ESI ${ }^{\dagger}$. In Fig. 3c, we compare the measured maximum back pressure for control pressures of approximately 6,9 , and 11 psi. A linear curve fit shows an $\mathrm{x}$-intercept of $\sim 4.5 \mathrm{psi}$, which is the minimum control pressure needed for the pump to be able to push fluid into a zero back pressure outlet. According to Fig. 3c, a control pressure of 9 psi is sufficient to generate a back pressure of approximately 4 psi, whereas a control pressure of 11 psi generates a back pressure of $\sim 8$ psi.

In Fig. $3 \mathrm{~d}$ we measure the flow rate as a function of the height of the outlet above the fluid source and pump. As expected, the flow rate slowly decreases with outlet height. An outlet height of $120 \mathrm{~cm}$ corresponds to $1.7 \mathrm{psi}$ of back pressure, in which case the average flow rate decreases from $19.3 \mu \mathrm{L} / \mathrm{min}$ to $17.2 \mu \mathrm{L} / \mathrm{min}$.

\subsection{D printed multiplexers and mixers}

We now use the example of a 3-to-2 multiplexer to illustrate how $3 \mathrm{D}$ printing enables high density 3D layout of microfluidic components in a single device. The simplest function of a 3-to-2 multiplexer is to pump fluid from any of three inputs to any of 2 outputs. As discussed in Section 3.2, a pump can be constructed with a DC placed between two valves. Therefore, the smallest component count for a 3-to-2 multiplexer is shown in Fig. 4a where a DC is placed between three input valves (V1, V2, V3) and two outlet
Table 2 Mixer timing logic. Red: actuated (pressure applied; valves closed); green: not actuated (valves open). Valves not listed in the table are closed.

\begin{tabular}{|c|c|c|c|c|c|}
\hline & $\mathrm{t}_{0}$ & $\mathrm{t}_{1}$ & $\mathrm{t}_{2}$ & $\mathrm{t}_{3}$ & $\mathrm{t}_{4}$ \\
\hline $\mathrm{V} 2$ & $\bullet$ & $\bullet$ & $\bullet$ & $\bullet$ & $\bullet$ \\
$\mathrm{V} 3$ & $\bullet$ & $\bullet$ & $\bullet$ & $\bullet$ & $\bullet$ \\
$\mathrm{DC}$ & $\bullet$ & $\bullet$ & $\bullet$ & $\bullet$ & $\bullet$ \\
$\mathrm{V} 5$ & $\bullet$ & $\bullet$ & $\bullet$ & $\bullet$ & $\bullet$ \\
\hline
\end{tabular}

valves (V4, V5). Pumping, for example, from Buffer to Outlet 1 uses V1-DC-V4 with all other valves closed. Similarly, pumping from Red to Outlet 2 uses V2-DC-V5. Fluid can be pumped from any of the inlets to any of the outlets by using the associated inlet and outlet valves in conjunction with the DC while keeping all other valves closed. (As a side note, the configuration in Fig. 4a can actually pump fluid from any inlet or outlet to any other inlet or outlet by using the corresponding valves with the DC and appropriate phasing of their actuations.)

One possible 3D layout of the components in Fig. 4a is shown in Fig. 4b in which V1, V2, and V3 are located directly above V4, DC, and V5, respectively. Flow channels and control channels are easily routed through $3 \mathrm{D}$ space to form the connections indicated in the schematic diagram in Fig. 4a. The valve, DC, and flow channel dimensions are the same as discussed in previous 

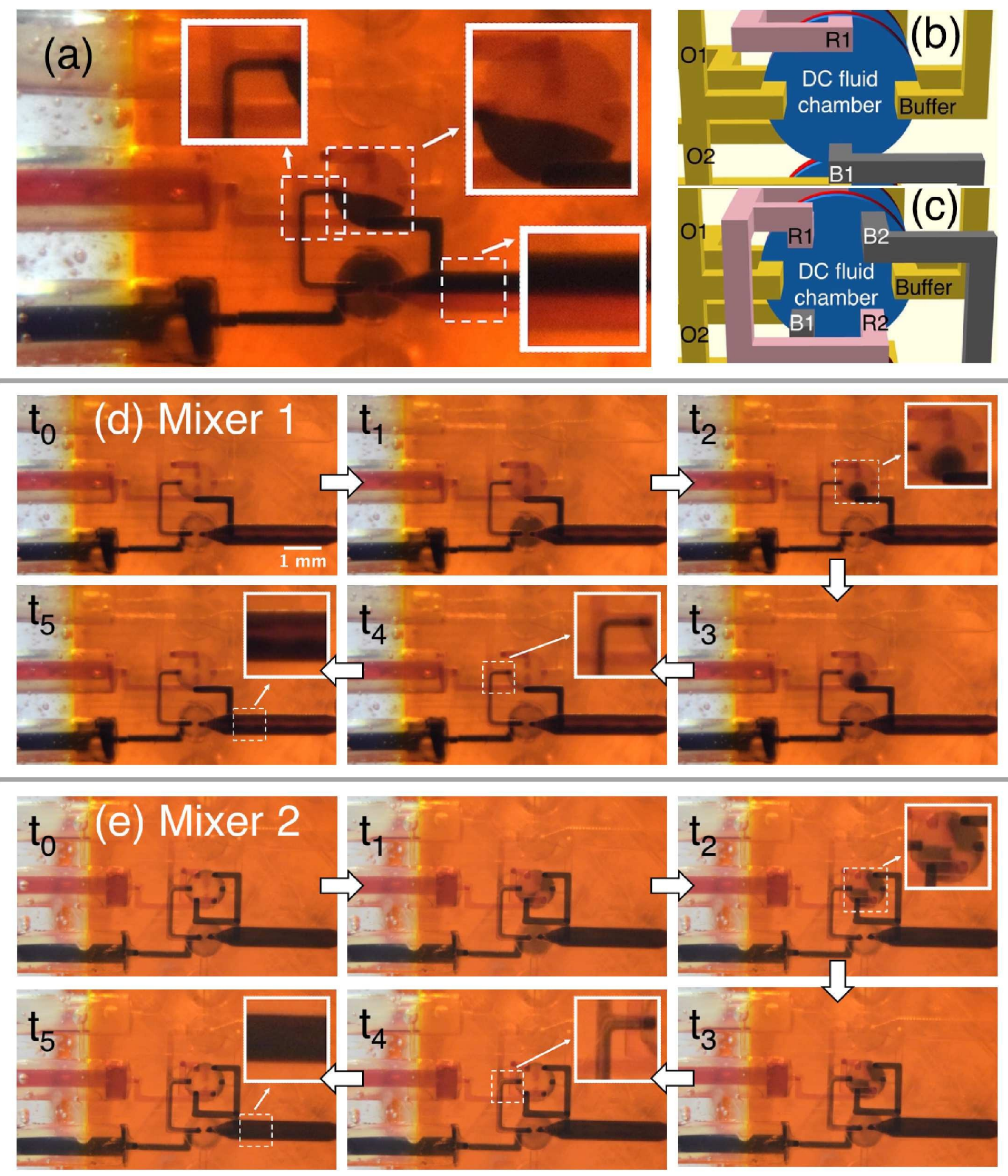

Fig. 5 (a) Flow generated only by gravity. Note lack of Red/Black mixing. (b, c) Bottom view of DC channel layout in (b) Mixer 1 and (c) Mixer 2 . Red channels, R1 in (b) and R1 and R2 in (c), connect the Red inlet valve (V2 in Fig. 4a) to the DC, while the black channels, B1 in (b) and B1 and B2 in (c), connect the Black inlet valve (V3) to the DC. Buffer is the channel that connects the Buffer inlet to the DC through valve V1. O1 and O2 connect the DC to Outlets 1 and 2, respectively, through valves V4 and V5. (d) and (e) compare the mixing performance of the designs in (b) and (c). See text for details. 
sections.

A fabricated device is shown in Fig. 4c, looking from below through the glass slide substrate. The valves V1, V2, and V3 are occluded (indicated by dashed white lines) behind V4, DC, and V5 (indicated by solid white lines). PTFE tubing is epoxied in the inlets on the left, and the outlet flow channels are on the right. The three inlet tubes contain buffer (Buffer), diluted red dye in water (Red), and diluted black dye in water (Black), respectively. Both Red and Black have previously been pumped through the device, followed by Buffer. This is the reason the flow channels from the Red and Black inlets to the DC are filled with Red and Black, respectively.

Figures $4 \mathrm{~d}-4 \mathrm{i}$ show an example set of operations conducted with the multiplexer that exercise the various combinations of inlets to outlets. It begins with Red being pumped to Outlet 1 (Fig. 4d), followed by Black to Outlet 2 (Fig. 4e), Buffer to Outlet 2 (Fig. 4f), Buffer to Outlet 1 (Fig. 4g), Red to Outlet 2 (Fig. 4h), and Black to Outlet 1 (Fig. 4i). Its dynamic operation is shown in ESI $\dagger$ Movie S1. During each inlet/outlet combination, the pump is typically run for 50 periods to more than fully flush the previous fluid in the large $(500 \mu \mathrm{m} \times 500 \mu \mathrm{m} \times 2.5 \mathrm{~mm})$ outlet channels. The large outlet channel size is chosen solely to make it easy to see the colored fluids. As a further note, it takes approximately 3 pump periods to flush fluid from the DC when switching from one fluid to another.

The multiplexer can also be used as a mixer by, for example, operating two of the inlet valves simultaneously during pumping, in which case the fluids from the two inlets will be drawn together through the pump and expelled into an outlet. Prior to initiating pump action, we first opened V2, V3, DC, and V5 while raising the reservoirs from which red and black fluid are drawn about $15 \mathrm{~cm}$ above the microfluidic device. All of the other valves are closed. Fig. 5a illustrates the gravity-induced flow of Red and Black through the device. The upper right inset shows Black entering the DC from below and Red from above, corresponding to the physical locations of their inlets into the DC. The upper left inset shows the segregated Red/Black flow stream through the DC outlet channel, which maintains its segregation through V5 and Outlet 2 (lower right inset image). Clearly, the only mixing that occurs is due to diffusion across the boundary between the two fluids.

Now consider simultaneous pumping from Red and Black into Outlet 2 according to the timing logic in Table 2. The results are shown in Fig. 5d in which each image shows the device state for the corresponding timing logic in Table 2 (note that $t_{5}$ is the same state as $t_{0}$ ). Prior to taking these images, the device was operated long enough such that it had reached a steady-state condition. At $\mathrm{t}_{1}$ fluid is draw into the DC through open valves V2 and V3, both of which are closed at $t_{2}$. The inset for $t_{2}$ shows the spatial segregation of fresh Red and Black just drawn into the DC. At $t_{3}$ the valve to Outlet 2, V5, is opened, following which fluid is expelled from the DC through V5 into Outlet 2 at $\mathrm{t}_{4}$. The inset at $\mathrm{t}_{4}$ shows similar Red/Black segregation in the DC outlet channel, but by the time it makes it through V5 and into Outlet 2 there is much more mixing than in Fig. 5a. However, there is still a discernible red streak near the middle of Outlet 2 (see inset at $\left.t_{5}\right)$.

As soon as we got this result we realized that mixing could be improved by increasing the degree to which Red and Black are interleaved in the DC, which is easily accomplished with a change in geometry. Consider for example the bottom view of the DC in Fig. $5 \mathrm{~b}$ in which Red is introduced into the DC through flow channel R1, and Black through B1. By splitting each Red and Black inlet into two inlets and interleaving them as shown in Fig. 5c (labeled as R1, R2, B1, and B2), additional mixing can be created in the DC. The mixing properties of the resultant device are shown in Fig. 5e using the same sequence of steps as Fig. 5d. The inset image for $t_{2}$ shows red and black regions localized around their respective DC inlets, while the inset at $t_{4}$ shows more Red/Black streams in the DC outlet channel, resulting in better mixing in Outlet 2 as seen in the inset at $t_{5}$. The rapid iteration time enabled by 3D printing allowed us to redesign, fabricate, and test this new DC inlet design within a day.

As a final comment, the 3-to-2 multiplexer in Fig. 4 can be readily scaled to larger numbers of inlets and outlets. At this point it is unclear what the practical scaling limit is, but it will likely be determined by the fabrication yield of the valves, in which case our fabrication techniques would need to be further refined to increase the valve yield.

\section{Summary}

In this paper we have demonstrated the potential of 3D printing to enable both rapid fabrication iteration and high density integration of microfluidic components. We have reported the smallest yet 3D printed valves and characterized valve performance and durability. Incorporation of a thermal initiator in the resin together with a post-print bake dramatically improves durability. Fifty two out of 54 valves were successfully tested up to 10,000 actuations, at which point we stopped the tests because of how long they took. One valve was tested to 1 million actuations, after which it still performed well. We have used these valves to create compact pumps and characterized their maximum back pressure and maximum flow rate. Flow rates as high as $40 \mu \mathrm{L} / \mathrm{min}$ have been demonstrated. We have also demonstrated a 3-to-2 multiplexer with integrated pump, and shown that it can also be used as a mixer. Moreover, we have shown the ability to implement and test a new idea to improve mixing within only a day, thereby illustrating the power of 3D printing to enable a "fail fast and often" iterative device development strategy.

\section{Acknowledgement}

We are grateful to the National Institutes of Health (R01 EB006124) for partial support of this work.

\section{References}

1 C. I. Rogers, K. Qaderi, A. T. Woolley and G. P. Nordin, Biomicrofluidics, 2015, 9, 1-9.

2 C. M. B. Ho, S. H. Ng, K. H. H. Li and Y.-J. Yoon, Lab Chip, 2015, 15, 3627-3637.

3 A. A. Yazdi, A. Popma, W. Wong, T. Nguyen, Y. Pan and J. Xu, Microfluid Nanofluid, 2016, 1-18. 
4 A. K. Au, W. Huynh, L. F. Horowitz and A. Folch, Angew. Chem., 2016, 128, 3926-3946.

5 D. Qin, Y. Xia and G. M. Whitesides, Adv. Mater., 1996, 8, 917-919.

6 D. C. Duffy, J. C. McDonald, O. J. A. Schueller and G. M. Whitesides, Anal. Chem., 1998, 70, 4974-4984.

7 J. C. McDonald, D. C. Duffy, J. R. Anderson, D. T. Chiu, H. Wu, O. J. Schueller and G. M. Whitesides, Electrophoresis, 2000, 21, 27-40.

8 H. Gong, M. Beauchamp, S. Perry, A. T. Woolley and G. P. Nordin, RSC Adv., 2015, 5, 106621-106632.

9 P. J. Kitson, M. H. Rosnes, V. Sans, V. Dragone and L. Cronin, Lab on a Chip, 2012, 12, 3267.

10 P. J. Kitson, M. D. Symes, V. Dragone and L. Cronin, Chem. Sci., 2013, 4, 3099.

11 K. C. Bhargava, B. Thompson and N. Malmstadt, PNAS, 2014, 111, 15013-15018.

12 A. I. Shallan, P. Smejkal, M. Corban, R. M. Guijt and M. C. Breadmore, Anal. Chem., 2014, 86, 3124-3130.

13 A. K. Au, W. Lee and A. Folch, Lab Chip, 2014, 14, 1294-1301.

14 A. K. Au, N. Bhattacharjee, L. F. Horowitz, T. C. Chang and A. Folch, Lab Chip, 2015, 15, 1934-1941.

15 R. D. Sochol, E. Sweet, C. C. Glick, S. Venkatesh, A. Avetisyan, K. F. Ekman, A. Raulinaitis, A. Tsai, A. Wienkers, K. Korner, K. Hanson, A. Long, B. J. Hightower, G. Slatton, D. C. Burnett, T. L. Massey, K. Iwai, L. P. Lee, K. S. J. Pister and L. Lin, Lab Chip, 2016, 16, 668-678.

16 C. I. Rogers, J. V. Pagaduan, G. P. Nordin and A. T. Woolley, Anal. Chem., 2011, 83, 6418-6425.

17 C. I. Rogers, J. B. Oxborrow, R. R. Anderson, L.-F. Tsai, G. P. Nordin and A. T. Woolley, Sens. Actuators B, 2014, 191, 438444.

18 G. Nordin, Design files for 3D printed microfluidic valve, pump, and multiplexer on figshare, CC-BY license, 2016, https: / / dx.doi.org/10.6084/m9.figshare.3219661.v1. 